\title{
IMF effect on sporadic-E layers at two northern polar cap sites: Part II - Electric field
}

\author{
T. Nygrén ${ }^{1}$, A. T. Aikio ${ }^{1}$, M. Voiculescu ${ }^{2,1}$, and J. M. Ruohoniemi ${ }^{3}$ \\ ${ }^{1}$ Department of Physical Sciences, University of Oulu, P.O. Box 3000, FIN-90014, Finland \\ ${ }^{2}$ Department of Physics, Faculty of Sciences, University "Dunărea de Jos" Galati, 800008 Galati, Romania \\ ${ }^{3}$ Applied Physics Laboratory, The Johns Hopkins University Applied Physics Laboratory, Laurel MD, 20723, USA
}

Received: 10 August 2006 - Revised: 6 February 2006 - Accepted: 13 March 2006 - Published: 19 May 2006

\begin{abstract}
This paper is the second in a series on a study of the link between IMF and sporadic-E layers within the polar cap. In Paper I (Voiculescu et al., 2006), an analysis of the sporadic-E data from Thule and Longyearbyen was presented. Here we concentrate on the electric field mechanism of sporadic-E generation. By means of model calculations we show that the mechanism is effective even at Thule, where the direction of the geomagnetic field departs from vertical only by $4^{\circ}$. The model calculations also lead to a revision of the electric field theory. Previously, a thin layer was assumed to grow at a convergent null in the vertical ion velocity, which is formed when the electric field points in the NW sector. Our calculations indicate that in the dynamic process of vertical plasma compression, a layer is generated at altitudes of high vertical convergence rather than at a null. Consequently, the layer generation is less sensitive than previously assumed to fluctuations of the electric field direction within the NW sector. The observed diurnal variations of sporadic-E occurrence at Longyearbyen and Thule are compared with the diurnal variations of the electric field, calculated using a representative range of IMF values by means of the statistical APL model. The results indicate that the main features of Es occurrence can be explained by the convection pattern controlled by the IMF. Electric fields calculated from the IMF observations are also used for producing distributions of sporadic-E occurrence as a function of electric field direction at the two sites. A marked difference between the distributions at Thule and Longyearbyen is found. A model estimate of the occurrence probability as a function of electric field direction is developed and a reasonable agreement between the model and the experimental occurrence is found. The calculation explains the differences between the distributions at the two sites in terms of the polar cap convection pattern. The conclusion is that the electric field is the ma-
\end{abstract}

Correspondence to: T. Nygrén

(tuomo.nygren@oulu.fi) jor cause for sporadic-E generation and, consequently, IMF has a clear control on the occurrence of sporadic $E$ within the polar cap.

Keywords. Ionosphere (Electric fields and currents; Plasma convection; Polar ionosphere)

\section{Introduction}

In the first paper of this series (Voiculescu et al., 2006; hereafter referred to as Paper I), the relation of the IMF and sporadic $\mathrm{E}$ at two polar cap sites, Longyearbyen and Thule, were studied. In this second paper, we investigate the same data in view of the electric field mechanism of layer generation.

The idea of sporadic-E generation by means of electric field dates back to a paper by Nygrén et al. (1984a), which suggests that provided the ionospheric electric field is strong enough and points in some direction in the (geomagnetic) NW sector, a thin layer of long-lived metallic ions may be created by vertical flow towards a convergent null in the vertical ion velocity profile. This conclusion was drawn by considering the final stationary state, where the vertical plasma compression is balanced by ambipolar diffusion at the flanks of the layer profile. This mechanism should only work at high latitudes, where electric fields of even tens of $\mathrm{mV} / \mathrm{m}$ are commonly encountered. This theory seems to be widely accepted (Bristow and Watkins, 1991; Bedey and Watkins, 2001; Kirkwood and Nilsson, 2000; Parkinson et al., 1998, 2001; Wan et al., 2001).

It was later found by Bristow and Watkins (1991) and Kirkwood and von Zahn $(1991,1992)$ that layers can be formed even when the field direction lies in the SW sector. Then there is no null in the vertical ion velocity profile, but the plasma flows downwards at all heights. In the lower E region, however, the faster ions at greater heights catch up to the slower ones below and therefore a slowly downward

Published by Copernicus GmbH on behalf of the European Geosciences Union. 
drifting layer can be generated. In this case the layer was expected to be thicker than in the case of the electric field pointing in the NW sector. It was also pointed out by Kirkwood and von Zahn $(1991,1992)$ that this mechanism should work even for field directions somewhat to the east of the south, because the lower part of the velocity profile is hardly altered when passing from the SW sector to the SE sector.

There is a basic difference between these two mechanisms. The first case corresponds to a stationary case when ions from a wide height range have finally reached a convergent null in the vertical ion velocity and the vertical drift is balanced by ambipolar diffusion. The second case deals with a more dynamic situation when layers are generated at heights where the convergence of the vertical ion velocity is positive (convergence is defined by $-d v_{z} / d z$, where $v_{z}$ is a vertical ion velocity and $z$ is altitude). Then the altitude of the layer is no more constant but the layer must drift slowly downwards (Kirkwood and von Zahn, 1991). According to these views, the generation mechanism should change abruptly if the electric field turns from the NW sector to SW sector. This also suggests that electric fields pointing slightly northwards and southwards of west might create layers at clearly different altitudes. Obviously this cannot happen, but the change must be continuous when the electric field passes the westward direction.

In this paper we show that in the dynamic process of vertical plasma compression, the layer is not necessarily created at a convergent null but rather the layer altitude is determined by the convergence of vertical ion velocity. This is true for all directions of the electric field. Provided the vertical velocity profile has a convergent null, the layer then drifts towards the null at a speed determined by the vertical ion velocity profile. A layer is created at the null only if the velocity convergence receives its maximum value at the null.

As seen in Paper I, the polar cap electric field may reach values of $20-30 \mathrm{mV} / \mathrm{m}$. This is certainly enough for a layer generation. However, the electric field mechanism breaks down at the magnetic pole where the geomagnetic field is vertical. Therefore, an obvious question is whether the tilt of the geomagnetic field is sufficient at Longyearbyen (dip angle $82^{\circ}$ ) and Thule (dip angle $86^{\circ}$ ) to make the mechanism effective. For example, MacDougall et al. (2000) claim that, due to the high inclination angle, the electric field mechanism is not very effective within the central polar cap. The simulations shown in this paper indicate that the mechanism is actually effective even at Thule, where the geomagnetic field direction departs from vertical only by $4^{\circ}$.

In Paper I, it is shown that the electric field direction calculated from the IMF at times of sporadic-E occurrence is mostly in agreement with the electric field theory. Here we show that at times when no layers or only few layers are observed, the polar cap electric field points in unfavourable directions. We also study the distributions of sporadic-E occurrence as a function of electric field direction. There is a marked difference between the distributions at Thule and at
Longyearbyen. The main features of this difference can be explained in terms of the polar cap convection patterns and the properties of the electric field mechanism.

\section{Model calculations of sporadic-E generation}

The vertical ion velocity is given by

$v_{z}=\frac{E \cos I}{B\left(1+\rho^{2}\right)}(\rho \cos \phi-\sin \phi)$,

where $E$ is the electric field, $B$ is the geomagnetic induction, $I$ is the dip angle, $\rho$ is the ratio of ion-neutral collision frequency and angular ion gyrofrequency, and $\phi$ is the angle between the electric field direction and the local geomagnetic north in the plane perpendicular to $B$, taken as positive from north to west (Nygrén et al., 1984a). This leads to a vertical convergence

$-\frac{d v_{z}}{d z}=\frac{E \cos I}{B\left(1+\rho^{2}\right)^{2}}\left[\left(\rho^{2}-1\right) \cos \phi-2 \rho \sin \phi\right] \frac{d \rho}{d z}$.

The convergence calculated from this formula is plotted in Fig. 1 at Thule and Longyearbyen for different heights and electric field directions. Since the convergence is proportional to the electric field, it is reasonable to normalise it by the electric field strength. The convergence is calculated for $\mathrm{Fe}^{+}$ions, and the ion neutral collision frequency is obtained from

$v_{i}=1.96 \times \frac{e}{m_{i}}\left(\frac{\alpha \mu}{\varepsilon_{0}}\right) N$

(see Nygrén et al., 1984b). Here $e$ is the positive elementary charge, $m_{i}$ is the ion mass, $\alpha=1.68 \cdot 10^{-30} \mathrm{~m}^{3}$ is the molecule polarizability, $\mu$ is the ion-neutral reduced mass, and $N$ is the neutral number density. For calculating the collision frequency, the MSISE-90 neutral atmospheric model at magnetic midnight in summer is adopted at each site. A weighted average is used for the neutral mass needed in calculating the reduced mass.

The continuous and dashed heavy lines in Fig. 1 indicate the altitudes of the convergent and divergent nulls, respectively. The altitude of a convergent null for each electric field direction is obtained from the condition $\rho=\tan \phi$ (Nygrén et al., 1984a). The vertical ion velocity is downwards between these two lines and upwards elsewhere.

Figure 1 shows enhanced positive velocity convergence, making a descending structure which already starts in the NE sector, goes all the way through the NW and SW sectors and ends in the SE sector. Within the SE sector the convergence decreases and drops to zero before the eastward field direction is reached. If the metal ions are originally evenly distributed, it is clear from the continuity equation that their density first starts growing at altitudes where the convergence is great. Hence, when we consider the dynamic process of layer generation, we can expect that the layer starts growing 

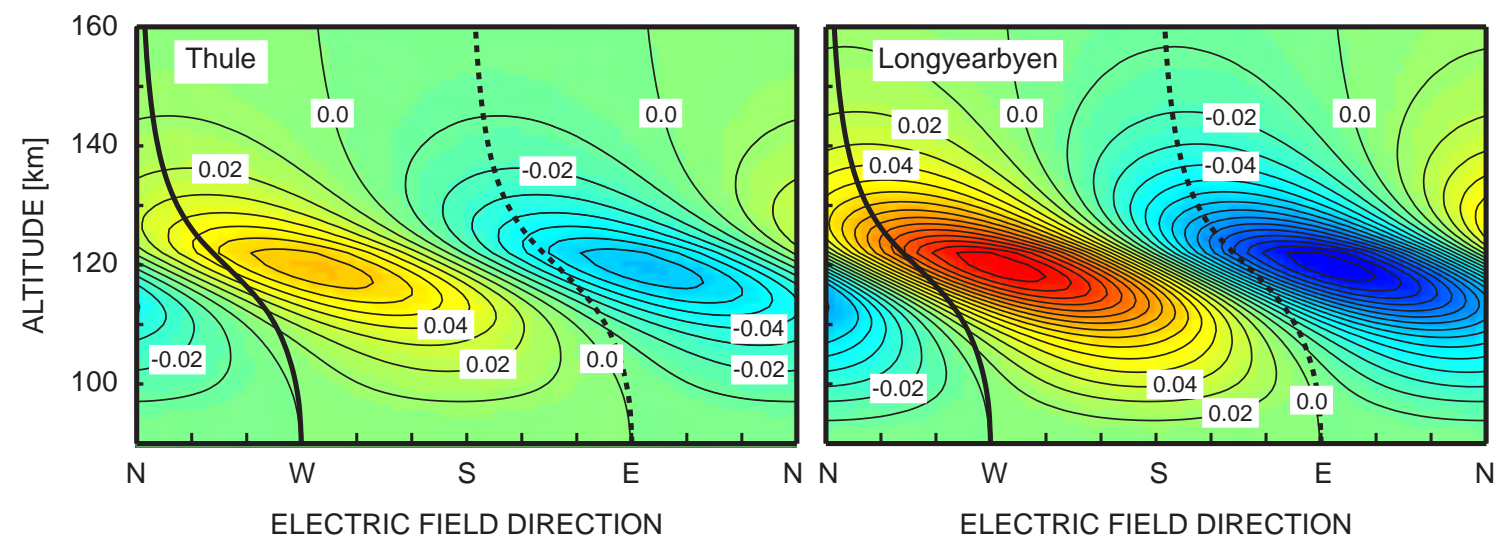

Fig. 1. Convergence of vertical velocity of $\mathrm{Fe}^{+}$ions for different electric field directions and altitudes at Thule and Longyearbyen. The convergence $-d v_{z} / d z$ is normalised by the electric field strength [leading to units $(\mathrm{m} /(\mathrm{Vs})$ ] and it is shown both in terms of colour and contour plot. MSISE-90 neutral atmospheric model is used at magnetic midnight in summer at each site. The continuous and dashed heavy lines indicate the altitudes of the convergent and divergent nulls, respectively.

close to the altitude of convergence maximum. The convergent null lies close to the convergence maximum only within a limited range of electric field directions. Thus we do not expect that a layer would start forming at the null for all field directions within the NW sector.

Figure 1 indicates that the layer formation is most effective for field directions around west; most preferably southward of west. This is in agreement with the observations by Kirkwood and von Zahn (1991). The altitude of layer formation decreases with increasing $\phi$. Figure 1 also reveals a possibility of layer formation for field directions eastward from south, which is in accordance to what was suggested by Kirkwood and von Zahn (1991). However, the layer formation must stop before the eastward direction.

In order to obtain a more detailed view of layer development, the growth of the layer was calculated by integrating the continuity equation. The electron density increment was calculated at 1-s steps, starting from a given initial ion density profile. The integration was made by means of the fourth-order Runge-Kutta method. The ion velocity needed in the continuity equation is given by the momentum equation. A constant electric field would give a velocity profile which does not depend on time. Due to the changing electron density profile, however, changes in the velocity profile are caused by ambipolar diffusion. Ambipolar diffusion is important at the flanks of the thin layer where steep density gradients are encountered. Notice that the layer growth will ultimately be stopped by ambipolar diffusion. This effect was taken into account in the calculations by making a correction, due to ambipolar diffusion, to the ion velocity profile after each integration step.

The growth of the layer at Thule for $\phi=60^{\circ}$ (the electric field in the NW quadrant) is shown in Fig. 2. The altitude of the convergent null for this field direction is indicated by a dashed line. A normalised density can be used since the

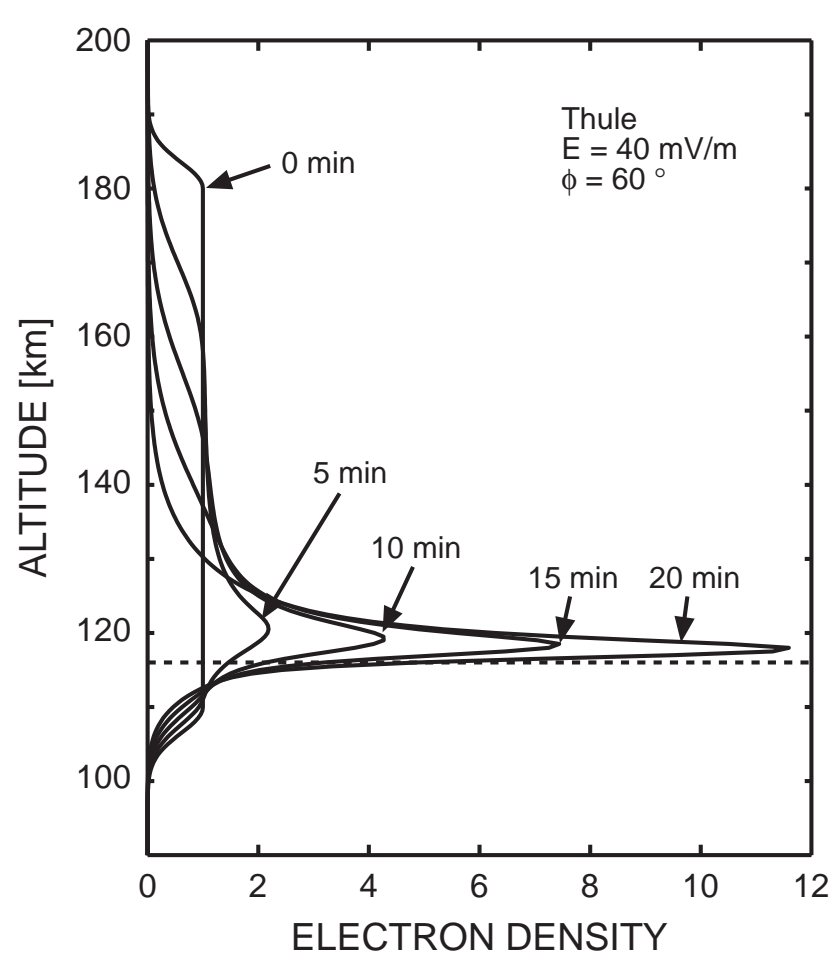

Fig. 2. Time development of layer generation at Thule at 5-min intervals. The initial $\mathrm{Fe}^{+}$ion density profile is denoted by $0 \mathrm{~min}$. The altitude of the convergent null in the vertical ion velocity profile is denoted by the dashed line.

continuity equation is linear in density. The details of layer growth would depend on the start profile of ion density. In the course of time, however, the solutions would converge towards the same final profile. Therefore, it is reasonable to use a simple start profile, since, after all, the density profile can have various shapes in the true ionosphere, depending on the 

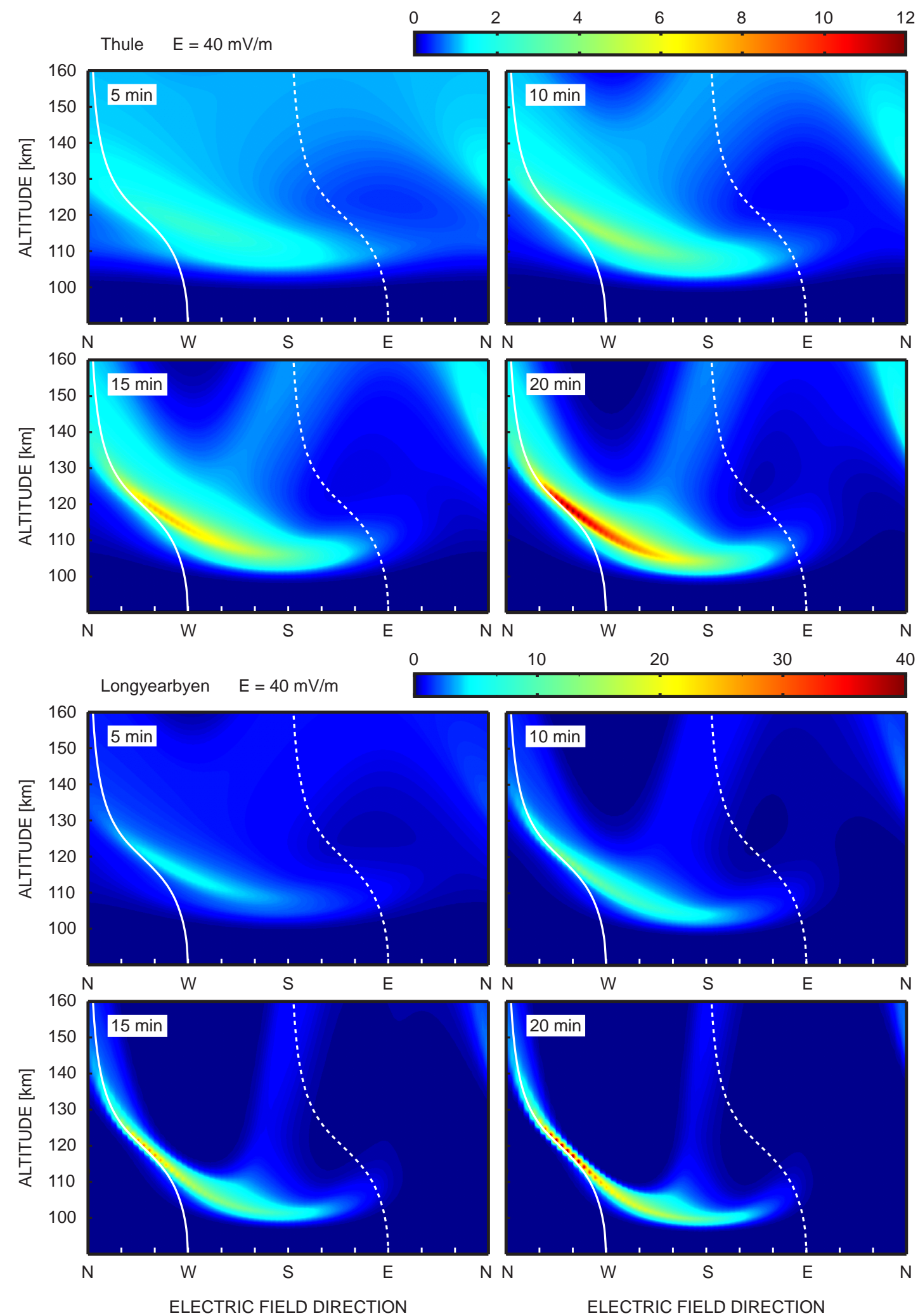

Fig. 3. Time development of $\mathrm{Fe}^{+}$layer generation for all electric field directions at Thule and Longyearbyen. The start ion density profile is the same as in Fig. 2. The colour bars indicate the scales of electron density. Convergent and divergent nulls are shown by the white continuous and dashed lines, respectively. 

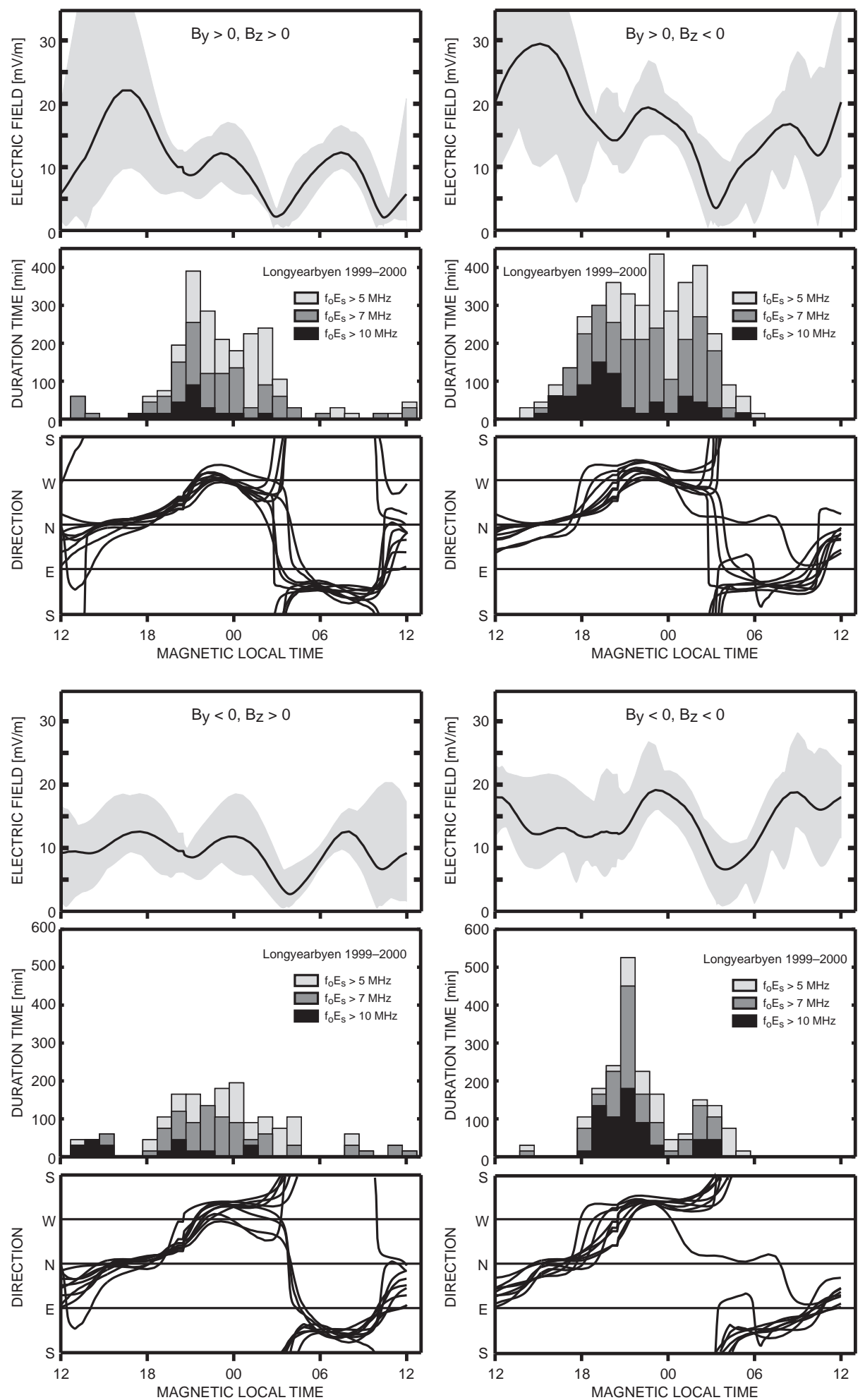

Fig. 4. Diurnal variations of electric field direction (bottom panels) and amplitude (top panels), calculated for different orientations of the IMF within each quadrant of the $B_{y} B_{z}$ plane, together with daily variations of sporadic-E occurrence observed at Longyearbyen in 19992000 (middle panels). The field direction is calculated from the APL convection model, using all directions of the IMF projection (the resolution of the model is $45^{\circ}$ ) and values 4,6 and $8 \mathrm{nT}$. The electric field directions are plotted by separate curves. The mean electric field amplitude is plotted by a continuous curve and the whole range of amplitude variation by grey shading. Separate Es distributions are plotted for layers with peak plasma frequencies higher than 5, 7 and $10 \mathrm{MHz}$. 
history of the plasma dynamics. Thus, the start density profile is taken as unity between 110 and $180 \mathrm{~km}$ with Gaussian flanks at the bottom and on the top. The electric field strength is $40 \mathrm{mV} / \mathrm{m}$. The result shows a substantial growth of a layer close to $120-\mathrm{km}$ altitude within a time of $20 \mathrm{~min}$. The peak density grows to more than a 10 -fold value from the original distribution. Most of the ions come from the upper part of the profile, which is due to a higher ion speed above the layer altitude. As predicted, the layer does not start forming at the null but at a greater altitude of maximum convergence above $120 \mathrm{~km}$ in height. The layer slowly drifts towards the null but has not yet reached it in $20 \mathrm{~min}$.

A similar calculation was made for all field directions with $5^{\circ}$ resolution. The results are shown in Fig. 3 for both for Thule and Longyearbyen. Layers always start forming at the regions of high vertical velocity convergence shown in Fig. 1. They are initially thick but, in the course of time, they become thinner while their peak density keeps growing. As predicted, the layer is not created at a convergent null of vertical ion velocity, except when the null lies close to the altitude of maximum convergence. The layer keeps drifting towards the null but it does not necessarily reach it in $20 \mathrm{~min}$. Most notably, the change from the NW sector to the SW sector takes place smoothly, so that the layer separates from the null before the westward direction is reached. At Thule the layer is thicker and weaker than at Longyearbyen, which is due to the smaller vertical ion velocity and the associated convergence. Another point is that at field directions slightly westwards of the north, the layer is weak and lies at high altitudes. This suggests that an observable layer is not created if the electric field direction is too close to north, even if the direction lies in the NW sector.

Figure 3 also shows that a layer is created at field directions somewhat eastwards of the south. These layers are at low altitudes. Within the SE sector a weaker, thick enhancement is encountered at greater heights, associated with a convergence in the upward velocity.

Figure 3 conclusively indicates that, theoretically, the polar electric field is capable of creating thin metal ion layers at both Thule and Longyearbyen. Of course, the true layer development depends on the field strength and the stability of its direction, as well as on the height distribution of the metal ions. Since the vertical velocity and the associated convergence are proportional to the electric field, and the layer growth rate is proportional to the vertical velocity, Fig. 3 also applies to other electric field strengths after the appropriate change in the time scale. The fact that a layer does not start forming at a convergent null has a further consequence: unlike that suggested by Wan et al. (2001), the layer formation is probably not very sensitive to fluctuations in the electric field direction within the NW sector.

\section{Daily variation of electric field}

Figures 4 and 5 are constructed to show the range of the diurnal variation of the ionospheric electric field direction and amplitude at Longyearbyen and Thule for various intensities and directions of the IMF projection in the $B_{y} B_{z}$ plane. In view of the distributions in Figs. 3 and 4 in Paper I, values 4, 6 and $8 \mathrm{nT}$ for the IMF projection can be considered to be representative for the present study. Using these values, the electric field is calculated by means of the APL model (Ruohoniemi and Greenwald, 1996) in $45^{\circ}$-steps of the IMF direction. A separate set of panels is presented for each quadrant of the $B_{y} B_{z}$ plane. The top panel in each set indicates the mean value of the electric field strength, together with the range of its variations. The middle panels show the diurnal distributions of the total sporadic-E duration from two years (1999-2000 for Longyearbyen and 1998-1999 for Thule). Finally, the set of curves in the bottom panels displays the variation of the electric field direction.

A typical feature of the electric field direction at Longyearbyen is that it contains step-like changes. In each of the four cases, the field direction is rather insensitive to the variation of the IMF direction and intensity. Figure 4 indicates that when layers are observed at Longyearbyen, the electric field points nearly always in a favourable direction. Also, at local times when no layers or only a few of them are observed, the electric field direction cannot be favourable in most cases. The step-like changes in the field direction seem to match the edges of the layer distributions with a reasonable accuracy. A recurrent feature in the electric field strength is a minimum occurring in the post-midnight sector. This minimum coincides with the morningside edge of the layer distributions and the related change in the field direction. The other variations of the field strength do not have any clear connections to the layer distributions. In particular, the electric field does not explain the nighttime minimum in Es distributions, which is visible when $B_{y}<0$ and $B_{z}<0$ and for intense layers when $B_{y}>0$ and $B_{z}<0$. Another unexplained feature is the high pre-midnight peak in the Es distribution observed for negative $B_{y}$ and $B_{z}$. These problems were already discussed in Paper I.

At Thule, the daily variation of the electric field direction is different. The field vector rotates more or less at constant speed throughout the day, which causes the descending curves seen in the bottom panels of Fig. 5. The phase of the electric field rotation is different in each of the four cases. This reflects the fact that the tilt angle of the polar cap convection pattern depends on the direction of the IMF. During its daily rotation, the electric field turns from the NW sector to the NE sector after midnight or early in the morning and passes south in the daytime or in the evening. It is obvious from the two cases with $B_{y}>0$ that the number of observed layers is small at times when the electric field points in the NE sector, also partly when it points in the SE sector or in directions close to north in the NW sector. Some indication of 

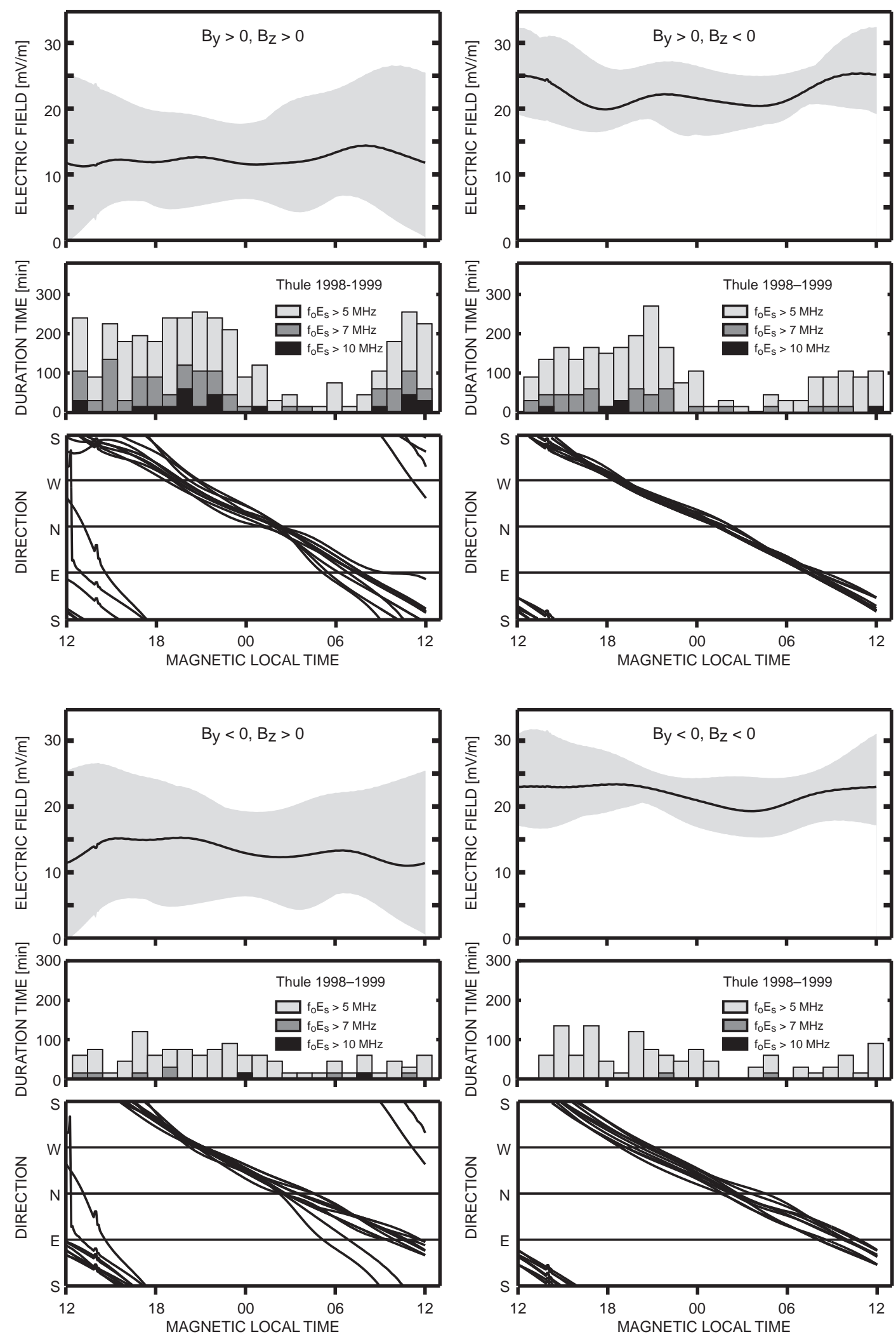

Fig. 5. Same as Fig. 4 for Thule in 1998-1999. 


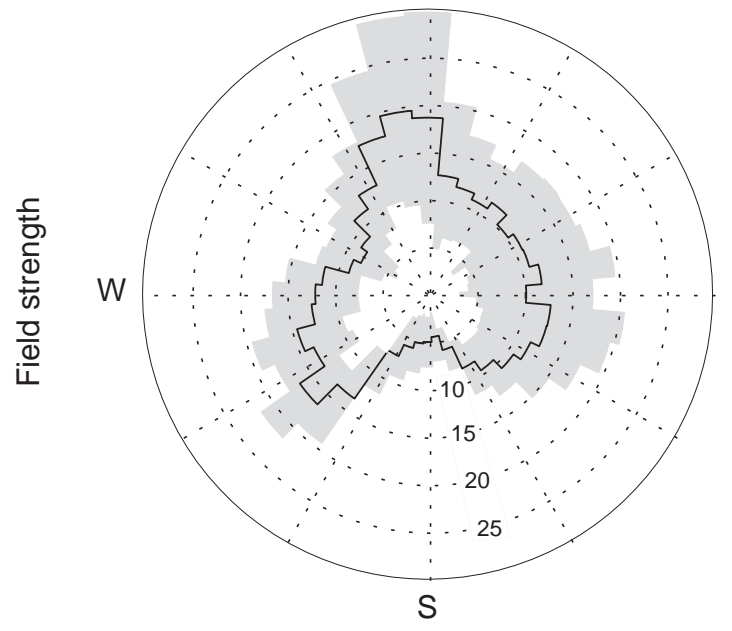

$\mathrm{N}$

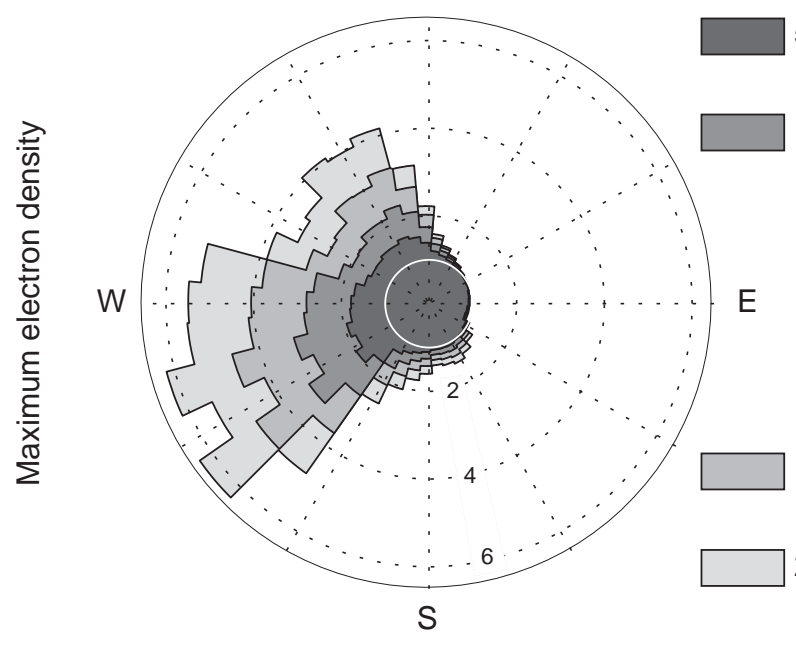

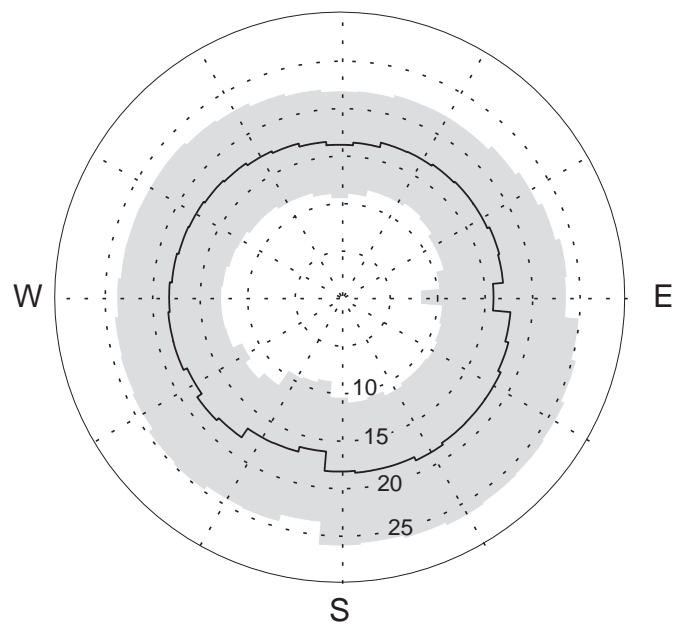

$\mathrm{N}$

$5 \min$

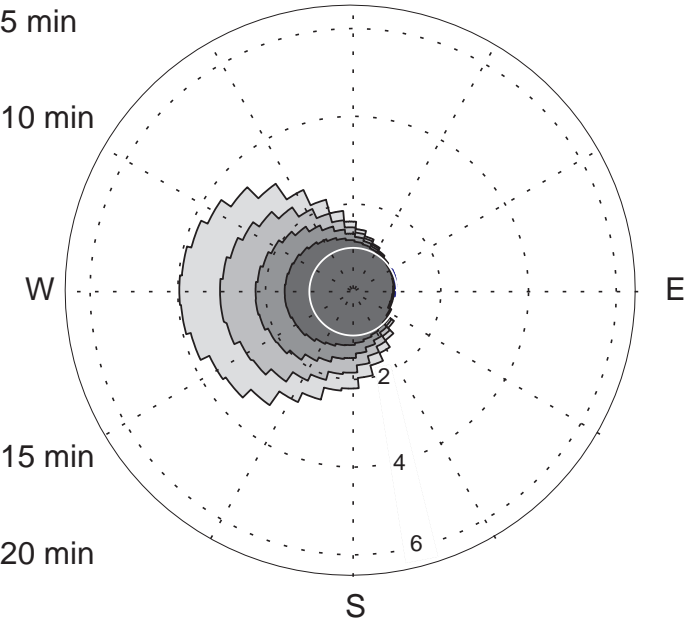

Fig. 6. Top panels: The average value of electric field intensity at Longyearbyen (left) and Thule (right) as a function of electric field direction. The electric field is calculated by means of the APL model using all directions of the IMF projection on the $B_{y} B_{z}$ plane and values 4, 6 and $8 \mathrm{nT}$. The shaded area indicates the standard deviation of the electric field intensity. The electric field direction is shown with respect to local geomagnetic north. Bottom panels: The peak values of calculated sporadic-E electron density, due to the average electric field, are shown in the top panels after 5-, 10-, 15- and 20-min compression. The start profile of the ion density is the same as in Fig. 1, and it is plotted by a white circle.

a similar behaviour is also seen when $B_{y}<0$, but this is less clearly visible because the total number of layers is small in these cases. The diurnal variation of the electric field amplitude is weak, and it cannot have any prominent effect on the Es distributions. The electric field is stronger when $B_{z}<0$ than in the opposite case, but this has no effect on the Es distributions either.

In conclusion, both at Longyearbyen and at Thule the electric field points in a direction favourable to the electric field mechanism at times when most of the layers are observed. This is true regardless of the orientation of the IMF. Also, layers are only rarely observed at times when the electric field points in unfavourable directions. This is all in good agreement with the electric field theory. A puzzling problem is the enhanced occurrence rate of Es layers during positive
$B_{y}$ and the weak preference of layers at Thule for positive $B_{z}$. This cannot be explained in terms of the electric field strength.

\section{Dependence of the sporadic-E occurrence on electric field direction}

A different way of presenting the behaviour of the electric field for all IMF conditions is to calculate the mean value of the electric field intensity for each electric field direction, when the IMF obtains all directions and values. This is portrayed for Longyearbyen and Thule in the top panels of Fig. 6, using the same APL model results as in Figs. 4 and 5. Here $10^{\circ}$-bins are used and the local geomagnetic north is 


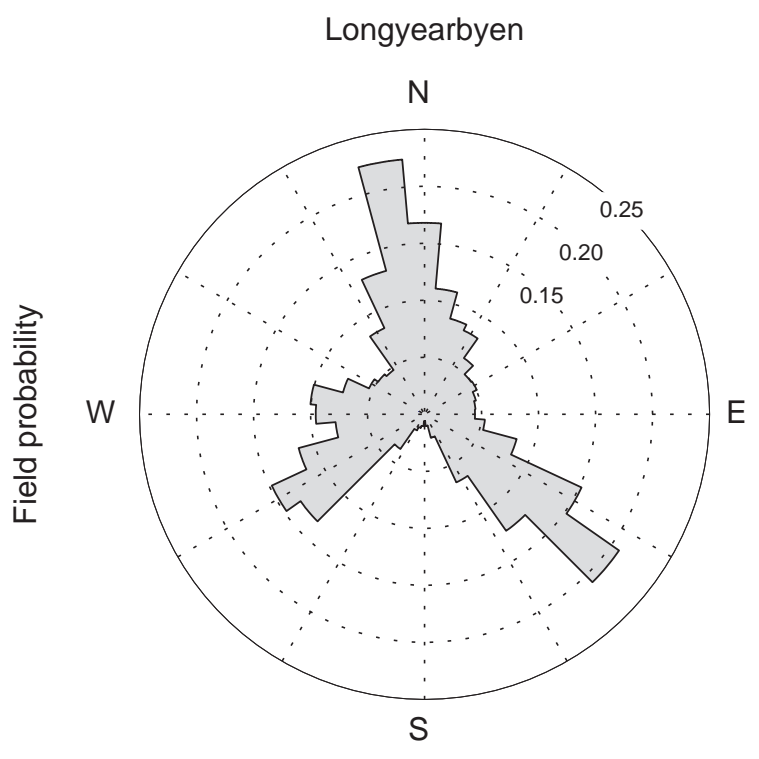

$\mathrm{N}$

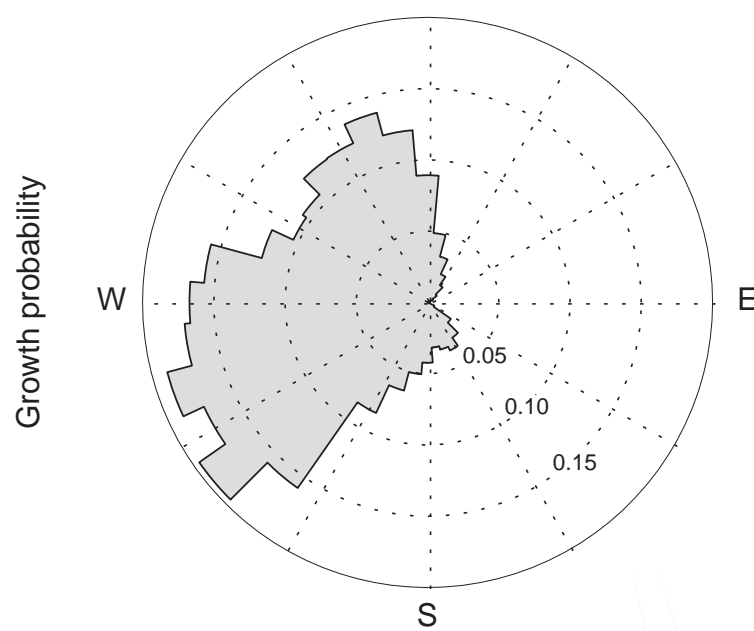

Thule
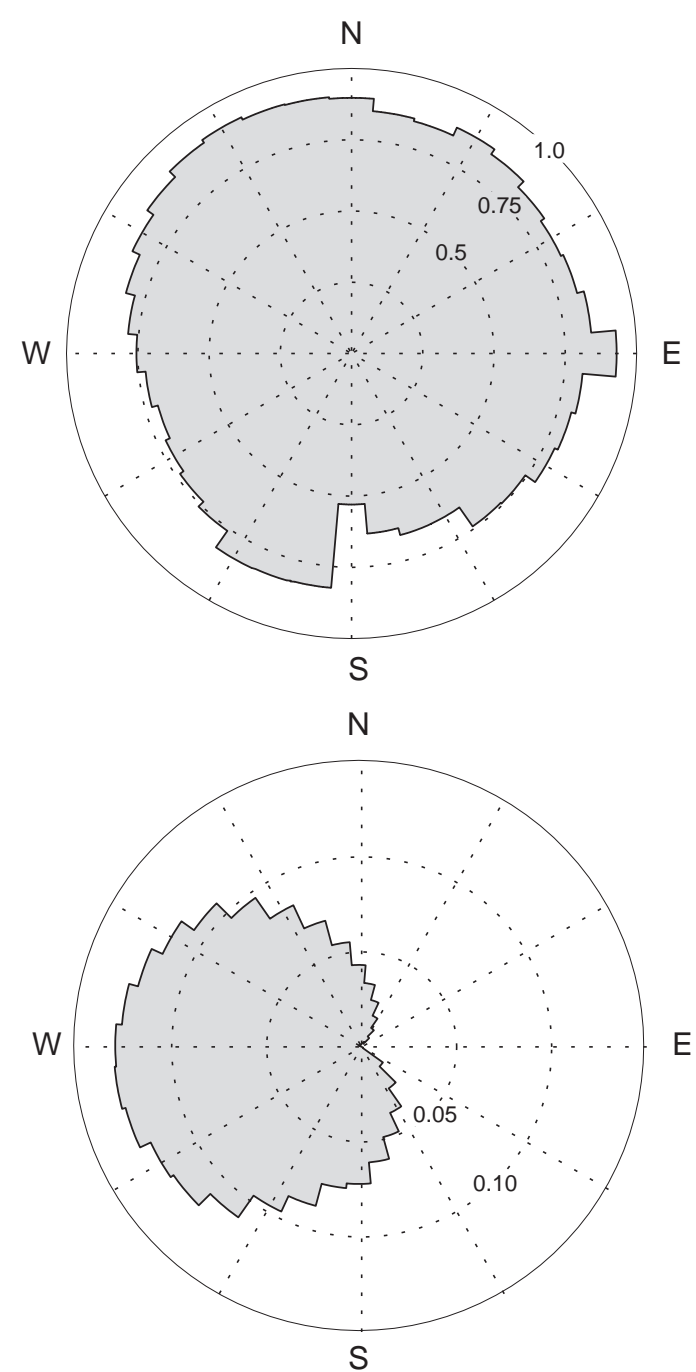

Fig. 7. Top panels: Unnormalised field probabilities for Longyearbyen (left) and Thule (right) for different directions of the electric field, calculated using the APL model. Bottom panels: Unnormalised growth probabilities for Longyearbyen and Thule, based on the layer growth shown in the bottom panels of Fig. 6. For definitions of "field probability" and "growth probability", as well as details of the calculation, see the text.

denoted by $\mathrm{N}$. The mean value is indicated by the continuous line and the standard deviation by the shaded region. A remarkable difference is seen between the distributions at the two sites; at Thule the distribution is fairly symmetric while at Longyearbyen it has three maxima separated roughly by $120^{\circ}$. Also, the field intensities are higher at Thule.

The bottom panels of Fig. 6 display the growth of Es layers when the electric field has the mean values shown in the top panels. The start value of the metal ion density is drawn by the white circle (the start profile is the same as in Fig. 1), and the peak electron density by different shadings $(5,10,15$ and $20 \mathrm{~min}$ after the electric field is turned on). Due to the fact that the electric field mechanism is ineffective within the NE sector and within a large part of the SE sector, the distribu- tions are necessarily asymmetric. At Longyearbyen the distributions have sharp edges, while a smooth directional variation is encountered at Thule. A remarkable fact at Longyearbyen is that intense layers are expected only within a limited sector covering parts of the NW and SW quadrants.

In addition to the diurnal variation of Es occurrence, it is of interest to investigate the distribution of Es occurrence as a function of electric field direction. Presumably, layers are more probably observed at field directions which produce the most intense layers.

Another factor which affects the probability is associated with the diurnal variation of the electric field direction. If the electric field points in a favourable direction for a long time, the probability of observing a layer with this field direction is 
great, whereas it is small in the opposite case. From Figs. 4 and 5 it is obvious that Longyearbyen and Thule are quite different in this respect. At Thule the field rotates continuously while at Longyearbyen its direction remains fairly stationary for extended time periods.

Next, we try to study the effect of this factor on the occurrence probability of sporadic E. Let us divide all possible directions of the ionospheric electric field into $n$ bins of equal width and all possible directions of the projection of the IMF in the $B_{y} B_{z}$ plane into $m$ bins of equal width. We first consider that the value of the IMF projection is fixed. The probability for the event $E_{i}$, meaning "electric field points into bin $i$ ", is $P\left(E_{i}\right)$ and, correspondingly, the probability for the event $B_{j}$, meaning "IMF points into bin $j$ ", is $P\left(B_{j}\right)$. For each event $E_{i}$, IMF always points into some bin, and therefore

$$
P\left(E_{i}\right)=\sum_{j=1}^{m} P\left(E_{i} \cap B_{j}\right) .
$$

Since the conditional probability of event $E_{i}$, when event $B_{j}$ is fixed, is $P\left(E_{i} \mid B_{j}\right)=P\left(E_{j} \cap B_{j}\right) / P\left(B_{j}\right)$, we obtain

$$
P\left(E_{i}\right)=\sum_{j=1}^{m} P\left(B_{j}\right) P\left(E_{i} \mid B_{j}\right)=\frac{1}{m} \sum_{j=1}^{m} P\left(E_{i} \mid B_{j}\right) .
$$

Here we have assumed that the direction of IMF in the $B_{y} B_{z}$ plane is evenly distributed, so that $P\left(B_{j}\right)=1 / m$ for all $j$. Next, we use the APL model to find all time intervals $\Delta t_{k}^{(i, j)}$, $k=1,2, \ldots$ within the whole day of $24 \mathrm{~h}$, when IMF is fixed to point into bin $j$ and the electric field points into bin $i$. This gives us an estimate of $P\left(E_{i} \mid B_{j}\right)$, i.e.

$P\left(E_{i} \mid B_{j}\right)=\frac{1}{24 \mathrm{~h}} \sum_{k} \Delta t_{k}^{(i, j)}$.

We can now calculate $P\left(E_{i}\right)$ from Eq. (5).

We adopt the same values of the IMF projection (4, 6 and $8 \mathrm{nT}$ ) as in Figs. 4, 5 and 6, and calculate separately the probabilities $P\left(E_{i}\right)$ in $10^{\circ}$-bins using Eqs. (6) and (5). The sum of these probabilities will be called "field probability" for convenience. Although these sums are not strictly the true probabilities of observing the electric field directions in the chosen sectors, they are still descriptive enough for the present purpose.

The field probabilities for Longyearbyen and Thule are plotted in the top panels of Fig. 7. The probabilities are quite different at the two sites. At Longyearbyen the field probability has three major peaks in the NW, SW and SE sectors. These are due to the fact that during the day, the electric field dwells for a long time around these directions. This was already visible in Fig. 4. At Thule the field probability is almost equal in all directions. This is due to the fact that the electric field turns around almost evenly throughout the day, as seen in Fig. 5.
Since the intense layers also grow rapidly, the occurrence probability connected to the intensity of the layer can be investigated by studying the growth rate. This is done using the calculations of layer growth shown in Fig. 6. An exponential fit is made to the time dependence of the peak electron density during the first ten minutes of compression when the effect of ambipolar diffusion is still rather small. The inverse of the resulting time constant is taken to be proportional to the associated probability, which will be called "growth probability" for convenience. The unscaled growth probability is shown in the bottom panels of Fig. 7.

As expected, the plots of growth probability resemble the plots of angular dependence of the peak density (bottom panels of Fig. 6). At Longyearbyen, high values extend roughly from north through west to southwest and the edges are very steep. At Thule, the highest values are also within the NW and SW sectors, but no steep edges are generated.

It is obvious that the probability of layer occurrence at a given electric field direction depends both on the dwell time of the electric field in this direction and on the efficiency of the compression. Hence, it is reasonable to define a "total probability" of layer occurrence as a product of the field and growth probabilities defined above. An unnormalised total probability for Longyearbyen and Thule is plotted in the top panels of Fig. 8.

The bottom panels of Fig. 8 show the experimental distributions of Es occurrence as a function of electric field direction for the whole time periods of collected data. The same $10^{\circ}$-bins are used as in the top panels. A clear difference between the two sites is seen. At Longyearbyen the distribution is strictly confined within a sector extending from the SW to the NW quadrant. When a layer is observed, the electric field has hardly ever an eastward component. The distributions are similar both for weak and intense layers. The highest occurrence probabilities take place at the southern edge of the distribution, making it very sharp. A secondary small maximum is encountered close to the northern edge of the distribution.

The distribution is broader at Thule, also extending to the SE sector. Even in this case the distribution has a maximum in the SW sector and a smaller secondary maximum in the NW sector. Another marked difference between Longyearbyen and Thule is that the distribution extends closer to the north at Longyearbyen than at Thule.

There is a considerable agreement between the modelled total probabilities in the top panels and the experimental occurrence rates in the bottom panels of Fig. 8. The main difference at Longyearbyen is that the maximum in the NW sector is much larger in the model probability. This can be understood when noticing that at field directions close to north, the layers are weak and lie at high altitudes. Therefore, they may easily remain unobserved in the upper $\mathrm{E}$ region and the bottomside $\mathrm{F}$ region. Another difference is that the steep edge in the SW sector lies closer to south in the total probability than in the occurrence rate. A most interesting feature is that 

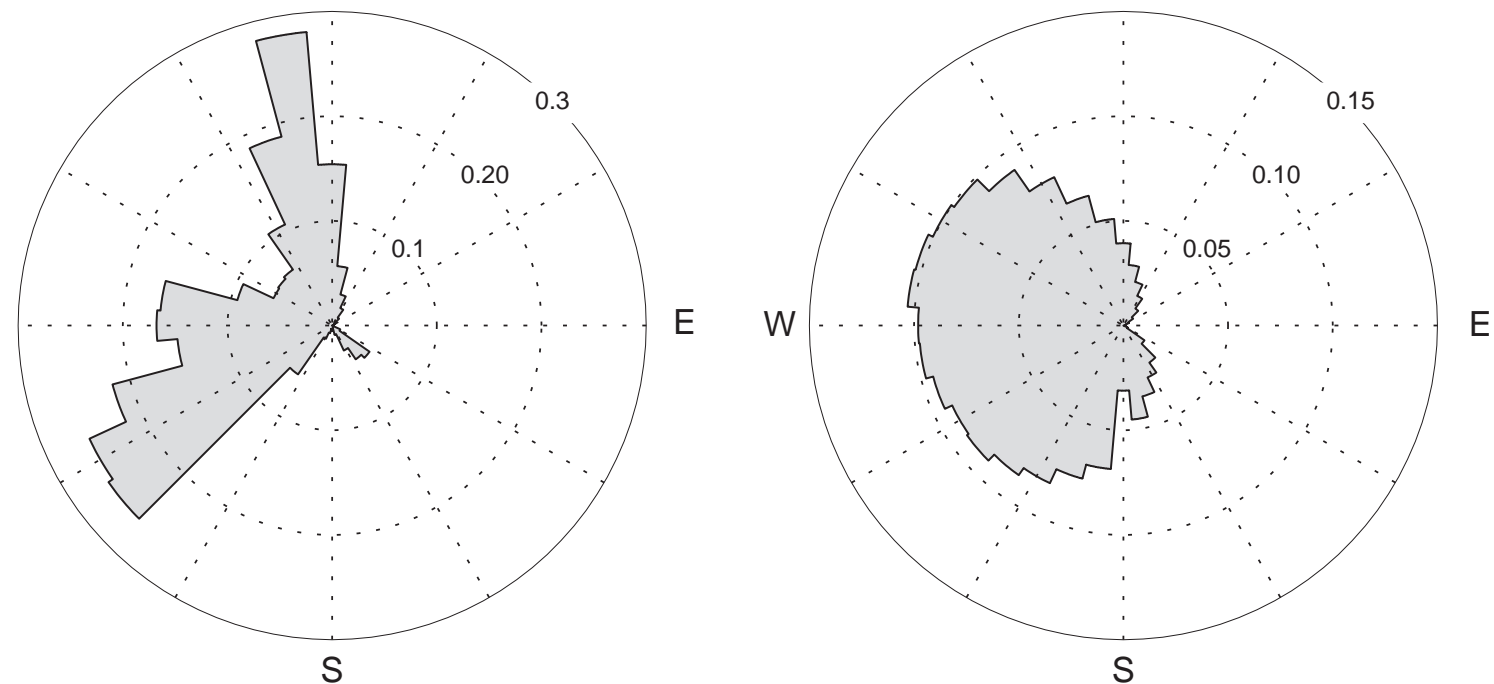

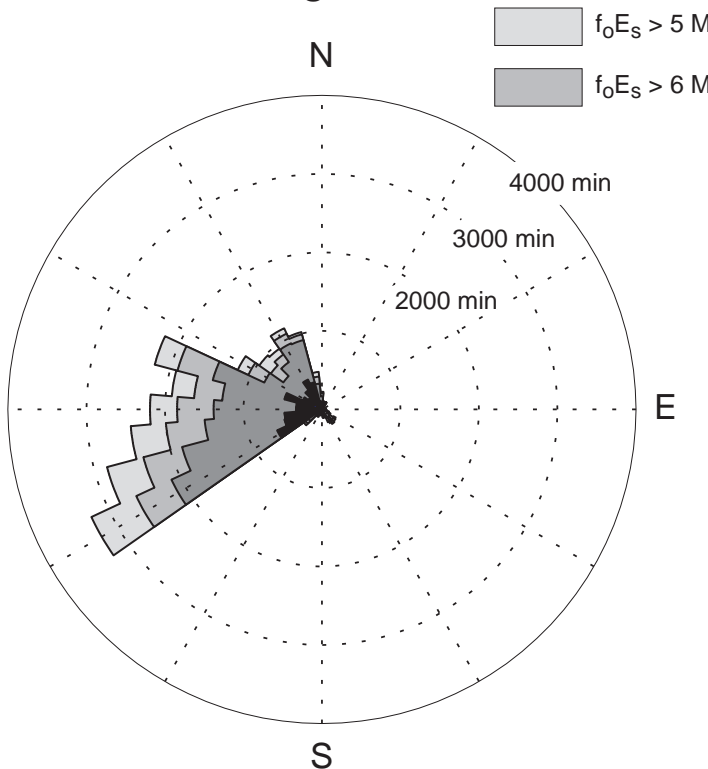

Longyearbyen, 1999-2000

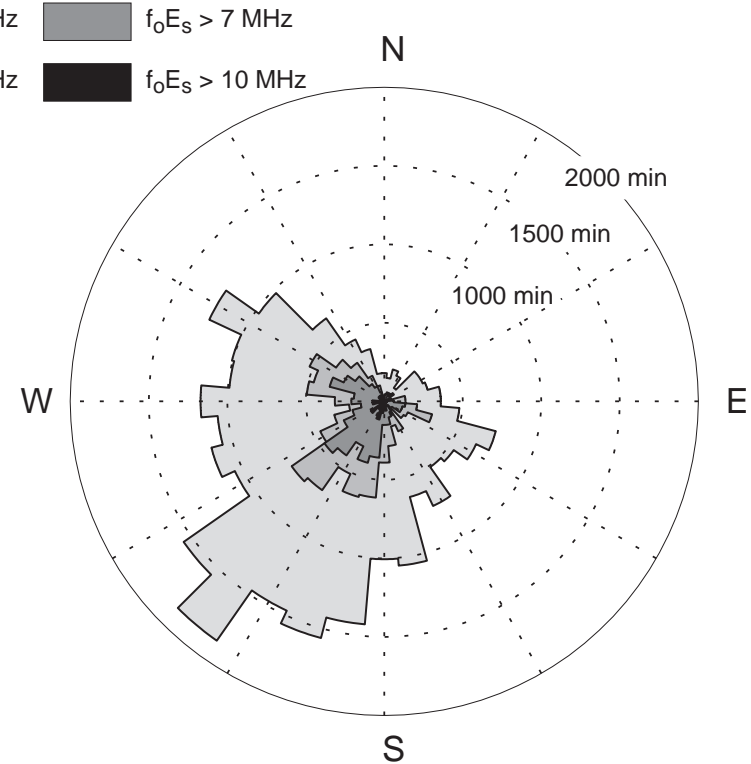

Thule, 1998-1999

Fig. 8. Top panels: The angular distribution of the unnormalised total probability of sporadic-E occurrence (product of the field and growth probabilities in Fig. 7) at Longyearbyen (left)and at Thule (right). Bottom panels: Distributions of observed Es occurrence at Longyearbyen and at Thule as a function of the electric field direction, calculated from the observed IMF using the APL model. Separate distributions are plotted for layers with peak plasma frequencies higher than 5, 6, 7 and $10 \mathrm{MHz}$.

both the total probability and the occurrence rate show a tiny peak in the SE sector.

At Thule, the occurrence rate is large roughly within a $180^{\circ}$ sector, extending from the NW quadrant to the SE quadrant via south. A clear maximum is observed in the SW sector. Similarly, the total probability is large within a $180^{\circ}$ sector, but there is roughly a $30^{\circ}$ difference in its orientation with respect to the occurrence rate. A second difference is that the total probability does not show a pronounced maximum as the occurrence rate does. At field directions close to north, layers are broader and weaker at Thule than at
Longyearbyen (see Figs. 3 and 6). Therefore, it is more likely that they remain unobserved in the upper $\mathrm{E}$ region and the bottomside $\mathrm{F}$ region. This may partly affect the fact that the northward boundary of experimental distribution lies closer to the north at Longyearbyen than at Thule.

\section{Summary and discussion}

In this paper the theory of Es generation by means of the ionospheric electric field is partly revised. It is shown that the layer always starts growing at an altitude of maximum 
convergence of vertical ion velocity. Hence, when the electric field points in the NW sector, the layer does not necessarily grow at the convergent null of the vertical ion velocity, as previously assumed. Then the layer generation is also not expected to be too sensitive to the variations of the electric field direction. In principle, a layer could be created (in the Northern Hemisphere) if the electric field points in any direction within a sector extending from local geomagnetic north via west, to some boundary eastwards of the south. In practice, however, field directions close to north or too much eastwards of the south produce weak and thick layers which are not necessarily observed as sporadic E. Also, electric field directions close to north produce layers at high altitudes, where they may be hard to observe in the bottomside $\mathrm{F}$ region.

The model calculations in Figs. 2 and 3 give decisive proof that sporadic-E layers can be created at very high latitudes by means of electric fields. At Thule the direction of the geomagnetic field vector departs from vertical only by $4^{\circ}$ and still the polar cap electric field is capable of collecting metal ions from the whole $\mathrm{E}$ region to a thin sheet in some tens of minutes.

A second purpose of this paper is to investigate the relation of polar cap electric field and experimental distributions of Es occurrence at two polar cap sites, Longyearbyen and Thule. Details of these data are explained in Paper I. The polar cap electric field is calculated for various projections of the IMF in the $B_{y} B_{z}$ plane. The statistical APL model (Ruohoniemi and Greenwald, 1996) is used for this purpose, and a set of IMF projections descriptive of the data set is applied in the calculations.

The diurnal variations of Es occurrence are different at the two sites, and the electric field directions, corresponding to the adopted range of the IMF projection, are mostly in good agreement with theory. However, there is a minimum in the occurrence around midnight at Longyearbyen, associated with southward IMF. This does not match well with the theory. In Paper I it was suggested that this may be due to fluctuating substorm-associated electric fields disturbing the layer generation at Longyearbyen.

Another minor disagreement is that at Thule, a small number of layers is also observed when the electric field points in unfavourable directions. This may be connected to the data set; it is possible that phenomena like polar showers and squalls are interpreted as sporadic $\mathrm{E}$ in data collection.

Although one might expect that the electric field strength has a straightforward effect on the Es occurrence, this does not seem to be so in the central polar cap. For instance, when IMF turns southwards and $B_{y}$ keeps positive, the electric field at Thule nearly doubles, but the occurrence of sporadic $\mathrm{E}$ decreases rather than grows. Hence, in addition to the electric field intensity and direction, some other factor must affect the layer occurrence. Since the IMF dependence of the layer occurrence is a clear experimental fact, this factor must be connected to the IMF. The observed discrepancies and their possible explanations are discussed more extensively in Paper I.

In addition to the diurnal variation, another view regarding the layer occurrence is offered by its dependence on the electric field direction. When the data are sorted in this manner, the results are in excellent agreement with the electric field theory. The resulting distributions from the two sites also contain features which need to be explained. In this paper a quantity describing the occurrence probability (in a somewhat loose sense) is defined. A remarkable similarity between the observed occurrence rate and theoretical occurrence probability is found. Possible reasons for the discrepancies are offered by the fact that the neutral wind is not taken into account in the calculations and, in some cases, layers may not be observed since they are embedded in the bottomside $\mathrm{F}$ layer.

In conclusion, the results of the present paper give conclusive proof of the determinative role of the electric field in sporadic-E generation within the polar cap. This is indicated by both the diurnal variations of the sporadic-E occurrence and the distribution of the occurrence as a function of the electric field direction. However, not all features in the observations can be explained by the simple electric field approach. This is not surprising, since the study is based on electric fields calculated by means of the statistical APL model. For more realistic research, true electric fields measured, for example, by coherent radars should be used. The present work also neglects the effect of neutral wind. A major problem is the lack of information on the availability and lack of horizontal dynamics of metal ions within the polar cap, which must have an effect on the sporadic-E occurrence. Still, it is clear that the IMF is able of controlling the generation of polar cap sporadic $\mathrm{E}$, which plays a part in radio-wave propagation and thus affects human activities within the polar regions. In this sense polar cap sporadic E can be considered as a manifestation of space weather.

Acknowledgements. We are grateful to T. Hyart and I. Virtanen for their work on data collection and preliminary data analysis. The Longyearbyen ionosonde is run by the University of Leicester. World Data Center C1 is acknowledged for the Thule ionosonde data (station code THJ77). Grant (project No 51465) from the Academy of Finland and support from Space Institute at the University of Oulu is also gratefully acknowledged.

Topical Editor M. Pinnock thanks two referees for their help in evaluating this paper.

\section{References}

Bedey, D. F., and Watkins, B. J.: Simultaneous observations of thin ion layers and the ionospheric electric field over Sondrestrom, J. Geophys. Res., 106(A5), 8169-8183, 2001.

Bristow, W. A. and Watkins, B. J.: Numerical simulation of the formation of thin ionization layers at high latitudes, Geophys. Res. Lett., 18, 404-407, 1991. 
Kirkwood, S. and Nilsson, H.: High-latitude sporadic-E and other thin layers - the role of magnetospheric electric fields, Space Sci. Rev., 91, 579-613, 2000.

Kirkwood, S. and von Zahn, U.: On the role of auroral electric fields in the formation of low altitude sporadic-E and sudden sodium layers, J. Atmos. Terr. Phys., 53, 389-407, 1991.

Kirkwood, S. and von Zahn, U.: The role of magnetospheric electric fields in producing auroral zone thin layers, Adv. Space Res., 12(10), 225-228, 1992.

MacDougall, J. W., Jayachandran, P. T., and Plane, J. M. C.: Polar cap sporadic E: part 1, Observations, J. Atmos. Solar-Terr. Phys., 62, 1155-1167, 2000.

Nygrén, T., Jalonen, L., Oksman, J., and Turunen, T.: The role of electric field and neutral wind direction in the formation of sporadic E layers, J. Atmos. Terr. Phys., 46, 373-381, 1984a.

Nygrén, T., Jalonen, L., Huuskonen, A., and Turunen, T.: Density profiles of sporadic E-layers containing two metal ion species, J. Atmos. Terr. Phys., 46, 885-893, 1984b.
Parkinson, M. L., Dyson, P. L., Monselesan, D. P., and Morris, R. J.: On the role of electric field direction in the formation of sporadic E-layers in the southern polar cap ionosphere, J. Atmos. SolarTerr. Phys., 60, 471-491, 1998.

Parkinson, M. L., Dyson, P. L., Monselesan, D. P., and Morris, R. J.: Formation of sporadic-E layers by magnetospheric electric fields in the southern polar cap ionosphere, Adv. Space Res., 27, 1399-1402, 2001.

Ruohoniemi, M. and Greenwald, R. A.: Statistical patterns of highlatitude convection obtained from Goose Bay HF radar observations, J. Geophys. Res., 101, 21 743-21 763, 1996.

Voiculescu, M., Aikio, A. T., Nygrén, T., and Ruohoniemi, J. M.: IMF effect on sporadic-E at two northern polar cap sites: Part I Statistical study, Ann. Geophys., 24, 887-900, 2006.

Wan, W., Liu, L., Parkinson, M. L., Liu, R., He, L., Breed, A. M., Dyson P. L., and Morris, R. J.: The effect of fluctuating ionospheric electric fields on Es-occurrence at cusp and polar cap latitudes, Adv. Space Res., 27, 1283-1288, 2001. 